\title{
Epigenetic mechanisms in cancer: push and pull between kneaded erasers and fate writers
}

REVIEW

\author{
This article was published in the following Dove Press journal: \\ International Journal of Nanomedicine \\ 24 April 2015 \\ Number of times this article has been viewed
}

\author{
Ammad Ahmad Farooqi \\ Jen-Yang Tang ${ }^{2-4}$ \\ Ruei-Nian $\mathrm{Li}^{5}$ \\ Muhammad Ismail' \\ Yung-Ting Chang ${ }^{6}$ \\ Chih-Wen Shu ${ }^{7}$ \\ Shyng-Shiou F Yuan ${ }^{8,9}$ \\ Jing-Ru Liu ${ }^{5}$ \\ Qaisar Mansoor \\ Chih-Jen Huang 2,3,* \\ Hsueh-Wei Chang,9-11,*
}

'Institute of Biomedical and Genetic Engineering (IBGE), KRL Hospital, Islamabad, Pakistan, ${ }^{2}$ Department of Radiation Oncology, Faculty of Medicine, College of Medicine, ${ }^{3}$ Department of Radiation Oncology, Kaohsiung Medical University Hospital, ${ }^{4}$ Department of

Radiation Oncology, Kaohsiung Municipal

Ta-Tung Hospital, ${ }^{5}$ Department of

Biomedical Science and Environmental

Biology, Kaohsiung Medical University,

Kaohsiung, Taiwan; ${ }^{6}$ Doctor Degree

Program in Marine Biotechnology, National

Sun Yat-sen University/Academia Sinica,

${ }^{7}$ Department of Medical Education and

Research, Kaohsiung Veterans General Hospital, ${ }^{8}$ Translational Research Center,

${ }^{9}$ Cancer Center, Kaohsiung Medical

University Hospital, ${ }^{10}$ Institute of Medical

Science and Technology, National Sun

Yat-sen University, ' Research Center of

Environmental Medicine, Kaohsiung Medical

University, Kaohsiung, Taiwan

*These authors contributed equally to this work

Correspondences: Chih-Jen Huang; Hsueh-Wei Chang

Department of Radiation Oncology, Kaohsiung Medical University Hospital; Department of

Biomedical Science and Environmental Biology,

Kaohsiung Medical University, Kaohsiung

80708, Taiwan

Tel +886 $73 \mid 2$ II 0 I ext 7| 24; 269 |

Email ccjjhh@kmu.edu.tw; changhw@kmu.

edu.tw

\begin{abstract}
Research concerning the epigenome over the years has systematically and sequentially shown substantial development and we have moved from global inhibition of modifications of the epigenome toward identification and targeted therapy against tumor-specific epigenetic mechanisms. In accordance with this approach, several drugs with epigenetically modulating activity have received considerable attention and appreciation, and recently emerging scientific evidence is uncovering details of their mode of action. High-throughput technologies have considerably improved our existing understanding of tumor suppressors, oncogenes, and signaling pathways that are key drivers of cancer. In this review, we summarize the general epigenetic mechanisms in cancer, including: the post-translational modification of DNA methyltransferase and its mediated inactivation of Ras association domain family 1 isoform A, Sonic hedgehog signaling, Wnt signaling, Notch signaling, transforming growth factor signaling, and natural products with epigenetic modification ability. Moreover, we introduce the importance of nanomedicine for delivery of natural products with modulating ability to epigenetic machinery in cancer cells. Such in-depth and comprehensive knowledge regarding epigenetic dysregulation will be helpful in the upcoming era of molecular genomic pathology for both detection and treatment of cancer. Epigenetic information will also be helpful when nanotherapy is used for epigenetic modification.
\end{abstract}

Keywords: epigenetic, modification, methylation, natural products, cancer

\section{Introduction}

The epigenome has been heralded as a key "missing piece" of the jigsaw puzzle for epigenetically understanding complex phenotypes, and a substantial amount of information has been added to the continuously broadening epigenetic landscape. Phenotypic variation commonly results from genetic mutation and environmental change. Using high-throughput technologies, scientists are able to capture the potentially dynamic interplay between DNA sequence factors and chromatin. The line between genetic and environmental variation is blurred by inherited epigenetic variation, which is sensitive to environmental input. Our concepts regarding epigenetic alterations in molecular oncology are rapidly expanding, and it is now known that active gene promoters are rich in $\mathrm{CpG}$ islands and normally lack DNA methylation. ${ }^{1}$ In active gene promoters, nucleosome-depleted regions occupy the upstream region of transcription start sites. ${ }^{2}$ These nucleosome-depleted regions are further flanked by nucleosomes marked by modified histones with trimethylated $\mathrm{H} 3$ on lysine 4 (H3K4me3). These nucleosomes had extensive acetylated lysine residues. ${ }^{3}$ The presence of the histone variant H2A.Z was found to be essential for destabilization of nucleosomes to facilitate initiation of transcription. Recently, non-CpG island promoters were shown to behave very similarly to $\mathrm{CpG}$ island promoters for epigenetic regulation involving DNA methylation, histone 
modification, and nucleosomal decoration. ${ }^{4}$ Experimental evidence is shedding light on the modulators that remove and/or add methyl marks to histones, pointing toward a new level of plasticity within this epigenetically modifiable system in the development and treatment of cancer. In this review, we summarize the general epigenetic mechanisms involved in cancer. This includes the network between DNA methyltransferase (DNMT), Ras association domain family 1 isoform A (RASSF1A), Sonic Hedgehog (SHH)/Wnt/ Notch/transforming growth factor (TGF) signaling, and natural products with epigenetic modification ability. Moreover, we introduce the concept of nanomedicine for the delivery of natural products with an ability to modulate the epigenetic machinery in cancer cells.

\section{Post-translational modification of DNMT/DNMT-mediated inactivation of RASSF I A}

DNMT, the enzyme responsible for catalyzing the transfer of a methyl group from $\mathrm{S}$-adenosylmethionine to cytosine, ${ }^{5}$ plays a vital role in maintaining genome stability. ${ }^{6}$ DNMTs were reported to be regulated by post-translational modifications, including citrullination (deamination) and phosphorylation. Citrullination, ie, the conversion of arginine into citrulline in a protein structure, within the DNA-binding site of histone $\mathrm{H} 1$ was reported to induce global chromatin decondensation and control the expression of some pluripotency genes. ${ }^{7}$ Protein citrullination upstream of the PWWP domain of wild-type DNMT3A may be mediated by peptidylarginine deiminase 4, but not the mutant form. ${ }^{8}$ Moreover, the half-life of DNMT3A was longer in cells expressing wild-type peptidylarginine deiminase 4 than in cells expressing the mutant form. The $\mathrm{v}$-Akt murine thymoma viral oncogene ( $A k t)$ has also been shown to stabilize and protect DNMT1 from degradation by phosphorylating it at Ser 143. ${ }^{9}$ DNMT3B was reported to be downregulated in $\mathrm{PC} 3$ prostate cancer cells by methylation and to inhibit cell proliferation and migration. ${ }^{10}$

\section{DNMT-mediated inactivation of RASSFIA}

RASSF1A is a potential tumor suppressor. ${ }^{11}$ Hypermethylation of the RASSF1A promoter has been reported in several cancers. ${ }^{12,13}$ Regulation of RASSF1A is thought to be mediated by DNMT proteins in responses to natural products and to viral infection. ${ }^{9}$ For example, mahanine, a plant-derived carbazole alkaloid, was reported to restore expression of RASSF1A in prostate cancer cells. Mechanistically, it was shown that mahanine induced DNMT3B and DNMT1 degradation and reduced nuclear accumulation of both these enzymes. Phosphorylation of Akt markedly reduced in mahanine-treated prostate cancer cells. Mahanine did not induce degradation of DNMT3B and DNMT1 in constitutively active Akt-expressing cells, suggesting that mahanine exerted inhibitory effects on wild-type Akt. ${ }^{9}$

Similarly, hepatitis B virus X protein (HBx; HBVgp3) negatively regulates RASSF1A. For example, HBx-positive cells markedly reduce RASSF1A expression and are associated with markedly higher expression of DNMT3B and DNMT1. HBx facilitated the positioning of DNMT1 in relation to the promoter of RASSF1A, as evidenced by chromatin immunoprecipitation analysis using DNMT1 antibody. ${ }^{14}$ Surprisingly, methylation changes in the RASSF1A gene were not significant in DNMT1 knockdown HBx cells. Therefore, the role of methylation in downregulation of $R A S S F 1 A$ remains controversial.

\section{Sonic Hedgehog signaling}

Hedgehog family proteins are important in embryonic development, ${ }^{15}$ and include three subgroups, ie, Desert Hedgehog, Indian Hedgehog, and $\mathrm{SHH} .{ }^{16}$ Among them, $\mathrm{SHH}$ is the best studied in terms of its regulation of methylation. Post-translational modifications are also commonly reported in histones in terms of regulation of methylation. ${ }^{17}$ It has recently reported that target genes of SHH induced intracellular signaling and are marked by an active $\mathrm{H} 3 \mathrm{~K} 4 \mathrm{me} 3$ mark and a repressive histone $\mathrm{H} 3 \mathrm{~K} 27 \mathrm{me} 3$ mark. ${ }^{18}$ Detailed analysis revealed that activation of $\mathrm{SHH}$ triggered switching of the epigenetic nanomachinery to an $\mathrm{H} 3 \mathrm{~K} 27 \mathrm{me} 3$ demethylase (lysine-specific demethylase 6B; KDM6B; Jmjd3)-centered coactivator complex from H3K27 methyltransferase, such as polycomb repressive complex $2 .{ }^{18}$ It was additionally noted that treatment with $\mathrm{SHH}$ triggered the formation of $\mathrm{H} 3 \mathrm{~K} 4$ methyltransferase complexes of KMT2F (SET domain containing 1A; SETD1A; Set1)/KMT2A (lysine (K)-specific methyltransferase 2A; MLL) positioning by Jmjd3 for resolution of the bivalent domain.

There is evidence that SHH signaling in neural stem cells and progenitor cells facilitates the transformation of tumor cells to heterogeneous states in a molecularly distinct but indistinguishable histological pattern. ${ }^{19}$ Tumor-initiating cells in different subtypes of the Patched (+/-) mouse model of spontaneous medulloblastoma were differentially distinct in terms of their molecular basis and also phenotypically as evidenced by differential activation of the Akt and extracellular regulated protein kinase pathways. Surprisingly, tumor-initiating cells from different subtypes had differential sensitivity to SHH pathway inhibitors. ${ }^{19}$ GLI1 is 
the immediate downstream activator of the SHH signaling pathway. ${ }^{20}$ In GLI1-silenced astrocytoma and medulloblastoma cell lines, Patched 1 expression was downregulated, suggesting that GLI1 mediates the regulation of Patched 1.

\section{Wnt signaling}

Wnt proteins form a family of highly conserved, secreted, lipid-modified signaling glycoproteins that regulate cell interactions during embryogenesis. Wnt signaling is important in cell proliferation and movement in the developing embryo. ${ }^{21}$ In adults, abnormal expression of Wnt signaling may lead to several diseases and cancers. ${ }^{21,22} \mathrm{Wnt}$ pathway regulators are methylated in colorectal cancer cells with increased DNMT1 expression. ${ }^{23}$ Epigenetic changes play a significant role in alteration of homeostasis of the epithelium, characterized by markedly enhanced proliferation, and development of cancer in normal adjacent tissue areas which are morphologically normal. ${ }^{24}$ Secreted frizzled-related protein 1 (SFRP1) mRNA expression in normal adjacent tissue samples was higher than that from colorectal cancer samples. ${ }^{25}$ When the crypts of normal adjacent tissue near the tumor site, the epithelial SFRP1 protein was decreased because of epigenetic silencing of the SFRP1 gene. Accordingly, the Wnt signaling pathways become potential targets in cancer therapy. ${ }^{26}$ Several proteins in Wnt signaling are introduced here.

\section{Wnt antagonists}

Several Wnt antagonists are reported to regulate Wnt signaling in the development of cancer. For example, SFRP1 is reported to induce apoptotic cell death by binding to Wnt-5 and Wnt-1 ligands, thus exerting inhibitory effects on Wnt receptor activation. ${ }^{25} \mathrm{CpG}$ methylation of Wnt antagonists such as SFRP5, SFRP1, SFRP2, Dickkopf Wnt signaling pathway inhibitor 2, Wnt inhibitory factor 1, wingless-type MMTV integration site family, member 3A, and SRY (sex determining region Y-box 17) were shown to significantly increase in the transition from normal tissues to adenoma. ${ }^{27}$ Moreover, Wnt inhibitory factor 1, SFRP1, SFRP2, and Dickkopf Wnt signaling pathway inhibitor 2 were hypermethylated during transition from adenoma to carcinoma. Therefore, stepwise selective hypermethylation of several Wnt antagonists increases the expression of Wnt target genes during development of colon carcinoma. ${ }^{27}$

Wnt signaling may be modulated by Wnt antagonists that interact with Wnt ligands themselves. For example, expression of SFRP5 and SFRP1 was markedly reduced in $\mathrm{HBX}$-infected cells. ${ }^{28}$ In vitro analysis confirmed the presence of hypermethylated promoter regions of SFRP1 and SFRP5 genes in $\mathrm{HBx}$-expressing hepatoma cells. Detailed assays provided evidence of $\mathrm{HBx}$-directed binding of DNMT3A and DNMT1 to the promoter regions of SFRP5 and SFRP1. Moreover, overexpression of SFRP5 and SFRP1 significantly inhibited Wnt-induced intracellular signaling and expression of target genes. Another Wnt antagonist, DKK1, is also reported to be involved in the progression of breast cancer. Polycomb protein chromobox homolog 7 (CBX7) effectively inhibits breast tumor initiation. ${ }^{29}$ Breast stem-like cells containing cell surface markers CD $44^{+} / \mathrm{CD} 24^{-} / \mathrm{ESA}^{+}$displayed downregulated expression of $\mathrm{CBX} 7$. CBX7 inhibited the Wnt/ $\beta$-catenin/T-cell factor pathway by enhancing expression of DKK1, a Wnt antagonist. ${ }^{29}$ Pharmacological inhibition of DKK1 in CBX7-overexpressing cells resulted in rescue of $\mathrm{CD}_{4}{ }^{+} / \mathrm{CD} 24^{-} / \mathrm{ESA}^{+}$cell populations and restoration of $\mathrm{Wnt}$ signaling. ${ }^{29}$ Dishevelled-binding antagonist of beta-catenin 1 (DACT1), an antagonist to $\mathrm{Wnt} / \beta$-catenin signaling, was reported to inhibit cancer cell proliferation, and is commonly downregulated in breast cancer. ${ }^{30}$ DACT1 and DACT2 are reported to bind efficiently to Dishevelled proteins. Cells reconstituted with DACT2 can inhibit proliferation, migration, and invasion of TPC-1 thyroid cancer cells. ${ }^{31}$

Wnt signaling plays important roles in embryonic development and tissue homeostasis via the transcription coactivator $\beta$-catenin. ${ }^{32}$ Modulating methylation may regulate $\beta$-catenin expression. For example, 5-aza-2'-deoxycytidine (5-aza-dC), a DNMT inhibitor, was reported to induce $\beta$-catenin expression in non-small cell lung cancer (NSCLC) cell lines. ${ }^{33}$ Dual luciferase assays revealed that, compared with Wnt3a-treated cells, treatment with 5-aza-dC did not exert significant effects on Wnt signaling activity, thus emphasizing the fact that $\beta$-catenin promoter methylation is a frequently noted mechanism in NSCLC. ${ }^{33}$ Accordingly, $\beta$-catenin is a potential epigenetic target in the treatment of NSCLC.

In addition to interaction with the Wnt ligand, Wnt signaling may also be modulated by Wnt antagonists that interact with Wnt receptors. Recently, the Wnt receptor was reported to be regulated epigenetically. For example, one of the essential components of SIN3 transcription regulator family member A-histone deacetylase (HDAC) co-repressor complex is BRMS1L (breast cancer metastasis suppressor 1 like) which was reported to inhibit target gene transcription. ${ }^{34}$ BRMS1L exerted its effects via epigenetic silencing of Wnt receptors, such as frizzled class receptor 10, through positioning of HDAC1 at the promoter and deacetylation of the histone H3K9. BRMS1L-silenced breast cancer cells in xenograft mice can promote the growth and metastasis of 
cancer. However, exogenous expression of BRMS1L markedly inhibited the metastasizing potential of cancer cells. ${ }^{34}$

\section{Tumor suppressors}

Some tumor suppressors are commonly inhibited epigenetically in cancer. For example, ephrin (receptor B3) is a tumor suppressor that is frequently downregulated in colorectal cancer. Mechanistically, the snail family zinc fingers 1 (SNAIL1; SNAI1) and 2 may compete with stem cell factors such as the achaete-scute family bHLH transcription factor 2 for binding to an E-box motif to repress ephrin (receptor B3) expression. ${ }^{35}$ SNAIL1 displaced achaete-scute family bHLH transcription factor 2 (an effector in the Wnt pathway), transcription factor 7-like 2 (T-cell-specific, HMG-box; TCF7L2) and E1A binding protein p300 to facilitate positioning of corepressor complexes consisting of the histone demethylase likes lysine (K)-specific demethylase $1 \mathrm{~A}$ and the HDACs. ${ }^{35}$ SOX10, a novel HMG-box-containing tumor suppressor, is reported to compete with transcription factor 4 to bind $\beta$-catenin to transrepress its target genes. ${ }^{36}$ It is epigenetically silenced in cancer cells. ${ }^{36}$ Enforced expression of SOX10 considerably inhibited the potential for metastasis and tumorigenicity. ${ }^{36}$ Additionally, SOX11 was reported as the tumor suppressor gene in nasopharyngeal carcinoma, and hypermethylation of its promoter induced cell proliferation and invasion. ${ }^{37}$

\section{microRNA}

Some microRNAs are epigenetically regulated in cancer cells. ${ }^{38}$ For example, BRMS1L is negatively regulated by miR-106b in breast cancer cells. ${ }^{34} \mathrm{Wnt}-7 \mathrm{~b}$ activates the Wnt- $\beta$-catenin pathway in oral squamous cell carcinoma, thus inducing the potential for proliferation and invasion. ${ }^{39}$ miR329 and miR410 are epigenetically silenced in cancer cells. Cotreatment with HDAC inhibitor and demethylation agent can restore the expressions of miR410 and miR329 in cancer cells. ${ }^{39}$ Betel nut alkaloid, namely arecoline, notably downregulated expression of miR410 and miR329, thus rescuing Wnt-7b induced signaling. ${ }^{39}$

\section{Notch signaling}

Ligand proteins binding to the extracellular domain of the receptor induce proteolytic cleavage and a release of the intracellular domain, which enters the cell nucleus to modify gene expression. Notch functions as a receptor for membrane-bound ligands to control hematopoietic stem cell proliferation and differentiation. ${ }^{40}$ The Notch pathway displays dual cellular function of oncogenic and tumor suppression, depending on its context. For example, Notch signaling is oncogenic in T-cells but tumor-suppressing in B-cell leukemia. ${ }^{40}$ Four Notch receptors (Notch 1-4) and five ligands, ie, Jagged (JAG) 1 and 2 and Delta-like ligand (DLL) 1,3 , and 4 , constitute the human Notch signaling.

Notch ligand signals through the Notch receptor, thus initiating the process of proteolytic cleavage by a disintegrin and metalloprotease domain (ADAM) of metalloprotease. ${ }^{41}$ JAG1 and DLL1 are ligands of the Notch 1 receptor with a notable role in transducing signals intracellularly. ${ }^{42}$ The truncated form of Notch is further proteolytically processed by $\gamma$-secretase within its transmembrane region to liberate the Notch intracellular domain, ${ }^{43}$ which undergoes nuclear accumulation and complexes with mastermind-like factor and DNA-binding protein RBPJ (recombination signal binding protein for immunoglobulin kappa $\mathrm{J}$; RВPJ $\kappa$ ) to form a Notch transcription complex. ${ }^{44}$ Mastermind-like factor promotes positioning of transcriptional coactivators and E1A binding protein p300 to trigger expression of Notch target genes. ${ }^{45,46}$ Alternatively, RBPJ may suppress transcription of target genes when it is not complexed with Notch proteins. ${ }^{47}$

\section{Notch 3 and Notch I}

Notch 3-silenced nasopharyngeal carcinoma cells show a marked increase in apoptotic cell death and significantly reduced growth. ${ }^{48} \mathrm{RBPJ}$ binding sites are present in the promoter of NFKB1 and Notch 3 modulates mRNA and protein expression of NFKB1 (p105/p50) in C666-1 cells, as evidenced by chromatin immunoprecipitation-quantitative polymerase chain reaction assay. Similarly, the chromatin regulator BORIS/CTCFL (CCCTC-binding factor [zinc finger protein]-like) is reported to be positioned at the Notch 3 promoter in cancer cells and efficiently induces and maintains the active chromatin state. ${ }^{49}$ Moreover, HDAC5 was reported to significantly increase gene expression of Notch 1 in glioma cell lines. ${ }^{50}$ Notch 1 was quantitatively regulated by miR-34 in prostate cancer cells. Treating epigenetically silenced miR-34 containing prostate cancer cells with BR-DIM, a formulated 3,3'-diindolylmethane, considerably enhanced expression of miR-34a. ${ }^{51}$

\section{JAGI and DLLI}

Cisplatin-resistant ovarian cancer cell lines showed upregulation of JAG1 expression because JAG1 was controlled by miR-199b-5p, but miR-199b-5p lost this control epigenetically. ${ }^{52}$ Intriguingly, drug-resistant ovarian cancer cells reconstituted with miR-199b-5p showed improved sensitivity to cisplatin-induced cytotoxicity. Likewise, 
JAG1-depleted ovarian cancer cells also showed sensitization to cisplatin-induced cytotoxicity ${ }^{52}$ Additionally, DLL1 expression is epigenetically silenced in gastric cancer cell lines. Treating cancer cells with 5-aza-dC may enhance DLL1 expression. ${ }^{48}$

\section{H4KI6ac}

It is interesting that activation of the Notch pathway markedly decreases the post-translational acetylation of histone $\mathrm{H} 4$ at lysine 16 (H4K16ac) in hepatoma cancer cells. ${ }^{53}$ NICD-silenced hepatoma SMMC7721 cells demonstrated an increase in $\mathrm{H} 4 \mathrm{~K} 16 \mathrm{ac}$, and similar results were obtained upon treating cancer cells with a Notch signaling pathway inhibitor. ${ }^{53}$ Detailed analysis revealed that the MDM2 protooncogene, E3 ubiquitin protein ligase, mediated the degradation of K (lysine) acetyltransferase 8 (MOF; KAT8). ${ }^{53}$

\section{RBPJ and HES}

Upon ligand binding, Notch receptors may be cleaved to release the intracellular domain, which translocates to the nucleus and associates with the RBPJ transcription factor. NICD can complex with MAML and RBPJ to form a Notch transcription complex ${ }^{44,54-56}$ and subsequently induces downstream transcriptional repressor genes such as the HES family bHLH transcription factor. Increased NICD expression with a consequent rise in HES1 expression was noted in nasopharyngeal cancer (SNU601) cells transfected with DLL1. ${ }^{48}$ Importantly, the active and nonmethylated HES5 locus in $\mathrm{T}$ acute lymphoblastic leukemia cells (T-ALL1) is rich in $\mathrm{H} 3 \mathrm{~K} 4 \mathrm{me} 3$ and $\mathrm{H} 3 \mathrm{~K} 9 \mathrm{Ac}$, but lacks H3K27me3 and $\mathrm{H} 3 \mathrm{~K} 9 \mathrm{me} 3 .{ }^{40}$ Moreover, the downregulated and nonnmethylated HES5 locus in other T-cells (Molt4) was downregulated at $\mathrm{H} 3 \mathrm{~K} 4 \mathrm{me} 3$ and deacetylated at $\mathrm{H} 3 \mathrm{~K} 9 \mathrm{Ac}$, but lacked H3K27me3 and H3K9me3. These results suggested that different HES5 activities leaded to different histone modifications. Therefore, tumor suppression in the Notch/HES pathway may become epigenetically silenced in acute lymphoblastic leukemia B-cells.

\section{Transforming growth factor signaling}

TGF- $\beta$ signaling may promote or inhibit tumor progression depending on the cellular context. ${ }^{57,58}$ Several proteins of the SMAD family and TGF- $\beta$ targets were reported to be involved in the TGF- $\beta$ signaling pathway. For example, decorin plays an antimetastatic role by antagonizing TGF$\beta$-induced signaling. The methylation status of the decorin $5^{\prime}$-UTR region and levels of the phosphorylated form of
SMAD 3 were notably higher in cancer cells with high metastatic potential. ${ }^{59}$ Treatment with the demethylating agent, 5-aza-dC, significantly reduced levels of phosphorylated SMAD 3 and enhanced DCN expression.

TGF- $\beta 1$ was reported to inhibit proliferation of normal ovarian epithelial cells, to induce nuclear translocation of SMAD 4, and to induce expression of ADAM metallopeptidase domain 19. However, this did not hold for TGF- $\beta 1$-refractory ovarian cancer cells. ${ }^{60}$ In-depth analysis identified repressive histone modifications (dimethyl-H3K9 and trimethyl-H3K27) and an HDAC-bound ADAM metallopeptidase domain 19 (promoter region). In T-cells deficient in SMAD 2 or SMAD 4, interleukin-9 expression was reported to be impaired, and was associated with enhanced EZH2 binding to the interleukin-9 locus and enrichment of repressive histone modification such as $\mathrm{H} 3 \mathrm{~K} 27$ trimethylation. ${ }^{61}$ F-box protein 32 , a TGF- $\beta /$ SMAD target gene, was noted to be epigenetically silenced in esophageal squamous cell carcinoma. ${ }^{62}$ Analysis of clinical specimens indicated that F-box protein 32 positively correlated with expression of SMAD 4 protein and expression of phosphorylated SMAD 2 and SMAD 3.

TGF- $\beta 1$ is also involved in regulating the proliferation of cancer stem cells. For example, TGF- $\beta 1$ induced expression of thrombospondin-1 in stem cell populations resembling mesenchymal stem cells. ${ }^{63}$ Repressive histone modification marks H3K27me2, H4K20me1, H3K9me1, and H3K9me2 were markedly suppressed in these stem cell populations. Moreover, methylation levels of H4K20, H3K9, and H3K27 in the thrombospondin-1 promoter region were notably enhanced in lysine (K)-specific demethylase 7A (KDM7A; JHDM1D)-silenced cells. ${ }^{63}$

Human dachshund homolog 1 (DACH1), a tumor suppressor, is epigenetically inactivated in different cancers. Hypermethylation of the DACH1 promoter was noted in hepatocellular carcinoma cells, and treatment with 5-aza-dC restored expression of DACH $1 .{ }^{64}$ Moreover, TGF- $\beta$ signaling was also involved in regulating the epithelial-mesenchymal transition. For example, expression of DACH1 inhibited epithelial-mesenchymal transition and metastasis by inhibiting TGF- $\beta$-induced signaling. ${ }^{65}$ Tumor size was reduced in xenografted mice with DACH1-expressing BGC823 cells. TGF- $\beta$-induced epithelial-mesenchymal transition was also noted in ovarian cancer HEYC2 cells, by increasing both expression and activity of DNMT1, DNMT3A, and DNMT3B. After treatment of DNMT inhibitor SGI-110 in ovarian cancer cells, TGF- $\beta$-induced epithelial-mesenchymal transition was impaired. ${ }^{66}$ 


\section{Natural products can mediate the epigenetic machinery}

Several natural products have been reported to have modulating effects on the epigenetic machinery. Such effects would be helpful in developing epigenetic-based treatments for cancer. For example, psammaplin A, isolated from Psammaplysilla sponge, improved radiation-induced cell destruction in astrocytoma U373MG and lung cancer A549 cell lines. ${ }^{67}$ Both DNMT3A and DNMT1 were depleted in U373MG and A549 cancer cells after treatment with either psammaplin A or zebularine.${ }^{67}$ Other natural products, including cyclopamine, curcumin, epigallocatechin 3-gallate, genistein, resveratrol, zerumbone, norcantharidin, and arsenic trioxide have been well reviewed for their ability to target SHH signaling. ${ }^{68}$

\section{Inhibitors of Wnt signaling}

Rabdoternin B and maoecrystal I, isolated from a natural ent-kauranoid library, were reported to inhibit the Wnt signaling pathway in a dose-responsive manner. ${ }^{69}$ These natural products also showed selective killing effects in several colon cancer cell lines but were less harmful to normal colon epithelial cell lines. Wnt signaling target genes, including c-Myc, cyclin D1, survivin, and Axin2, were also downregulated by maoecrystal I. Additionally, magnolol, a neolignan from the cortex of Magnolia obovata, was reported to inhibit Wnt3ainduced $\beta$-catenin translocation and its downstream target gene expression. ${ }^{70}$

Natural products involving TGF- $\beta$ signaling in the treatment of cancer has also been well reviewed. ${ }^{71}$ Additionally, saponins from the roots of Platycodon grandiflorum notably inhibited TGF- $\beta 1$-induced p-SMAD $2 / 3$ levels and increased the expression of SMAD 7 in lung carcinoma A549 cells. ${ }^{72}$ Corilagin, a major active component of Phyllanthus niruri L., a medicinal herb, was reported to have hepatoprotective, antiviral, and anticancer activity. ${ }^{73}$ These investigators found that secretion of TGF- $\beta$ in ovarian cancer cell lines was specifically inhibited by corilagin.

\section{HDAC inhibitors}

Some natural products may target HDAC, and serve as potential HDAC inhibitors. For example, epigallocatechin 3 -gallate triggered upregulation of the Raf kinase inhibitor protein by inhibiting HDAC activity, which further increased the expression of histone H3. ${ }^{74}$ Moreover, epigallocatechin 3 -gallate also effectively inhibited the activity of matrix metalloproteinase- 2 and matrix metalloproteinase- 9 , nuclear translocation of nuclear factor kappa B, and Matrigel invasion in AsPC-1 cells. ${ }^{74}$ Genistein, an epigallocatechin 3-gallate herbal alkenylbenzene methyleugenol-derived oxidative metabolite, efficiently exerted inhibitory effects on HDAC1 activity. ${ }^{75}$ Genistein was also reported to induce expression of DKK1 by acetylation of histone $\mathrm{H} 3$ at the DKK1 promoter region in HCT15 and SW480 cells. However, DNA methylation at the promoter region of DKK1 was not modulated by genistein in colon cancer cells. ${ }^{76}$ Psammaplin A (11c), a marine metabolite, was noted to be highly effective against HDAC 1.77

\section{Cell cycle control genes}

Methylation of cell cycle control genes can also be modulated by natural products. For example, an aqueous extract of Opuntia ficus indica, and its bioactive ingredient, betalain indicaxanthin, reportedly induced demethylation of cyclindependent kinase inhibitor 2A (p16; INK4A; CDKN2A) which is a tumor suppressor gene. ${ }^{78}$ Similarly, methylation of the oxidative stress-related gene was modulated by a natural product. For example, Z-ligustilide, isolated from the medicinal herb Radix Angelicae sinensis (RAS), efficiently restored expression of the nuclear factor erythroid 2-like 2 in murine prostate cancer (TRAMP C1) cells. ${ }^{79}$ The methylation level of the first five CpGs of the promoter of erythroid 2-like 2 was remarkably reduced upon treatment with Z-ligustilide and RAS, as evidenced by bisulfite genomic sequencing. DNMT activity was also suppressed in cells treated with Z-ligustilide and RAS. ${ }^{79}$

\section{Nanotechnological approaches to modulate epigenetic machinery in cancer cells}

Improving the bioavailability and delivery of synthetic compounds and natural products to the target site is a rapidly growing area of research. Accumulating evidence shows that nanotechnological delivery has been applied to cancer therapy. For example, transcription factor AP-2 epsilon is noted to be epigenetically repressed in gastric cancer cells, and consequently decreased the responsiveness of cancer cells to 5-fluorouridine. 5-aza-dC and 5-fluorouridine may be encapsulated to deliver nanotechnologically by gelatinasestimuli nanoparticles and the response of gastric cancer cells to 5 -fluorouridine is enhanced ${ }^{80}$ Lipid-polymer nanoparticles encapsulating 5-aza-dC and doxorubicin were noted to be efficiently internalized by breast cancer (MDA-MB231) cells, thus improving drug uptake and drug-induced cell death. ${ }^{81}$ Mitoxantrone-loaded anacardic acid-containing liposomes 
were reported to significantly induce apoptosis in melanoma (A375) cells. ${ }^{82}$ Metallofullerenol nanoparticles were applied to epigenetic modulation of human breast cancer (MCF-7) cells for both in vitro and in vivo analysis. ${ }^{83}$

Combination treatment with epigenetic-targeted and chemotherapeutic drugs may generate synergistic effect for cancer therapy. For example, decitabine, a DNA hypermethylation inhibitor, effectively enhanced the chemotherapeutic response to doxorubicin. ${ }^{84}$ After the combined treatment with nanoparticles loaded low-dose decitabine or doxorubicin, the proportion of cancer stem cells with high aldehyde dehydrogenase activity in the mammospheres of breast cancer (MDA-MB-231) cells were marked reduced. Systemically delivered decitabineloaded nanoparticles considerably reduced DNMT1 and DNMT3b levels in mice xenografted with MB-MDA-231 cells. $^{84}$ Therefore, nanotechnology is helpful in the delivery of natural products for improved cancer therapy.

\section{Conclusion}

In this review, we have summarized general epigenetic mechanisms in cancer therapeutic drugs and explained their complex networking. We have also discussed and critically analyzed a number of natural products with epigenetic modifying ability. Recent nanotechnological advances indicate that nanoparticle-assisted delivery markedly improves the bioavailability of natural products with epigenetic modulation ability at the target site. There is a rapidly expanding list of synthetic and natural products with considerable anticancer and epigenetic modifying activity. In line with this approach, nanostructured materials, including nanoemulsions, nanoparticles, and nanotubules, have shown potential as effective carriers of epigenetic machinery targeting agents. This deeper and comprehensive knowledge regarding epigenetic modification and improvement of nanodelivery systems will be helpful in the treatment of cancer.

\section{Acknowledgments}

This work was supported by funds from the Ministry of Science and Technology (103-2320-B-037-008), the Kaohsiung Medical University "Aim for the Top Universities Grant" (KMU-TP103A33), the National Sun Yat-sen University-KMU Joint Research Project (NSYSU-KMU 104-p036), the Kaohsiung Municipal Ta-Tung Hospital (kmtth-102-011), the Health and Welfare Surcharge of Tobacco Products, the Ministry of Health and Welfare, Taiwan, Republic of China (MOHW104-TDU-B-212-124003, MOHW103-TDU-212-114007), and CheMei-KMU Joint Project (103CM-KMU-09). We also thank Hans-Uwe Dahms for assistance with English editing.

\section{Disclosure}

The authors report no conflicts of interest in this work.

\section{References}

1. Jones PA. Functions of DNA methylation: islands, start sites, gene bodies and beyond. Nat Rev Genet. 2012;13(7):484-492.

2. Bai L, Ondracka A, Cross FR. Multiple sequence-specific factors generate the nucleosome-depleted region on CLN2 promoter. Mol Cell. 2011;42(4):465-476.

3. Baylin SB, Jones PA. A decade of exploring the cancer epigenome biological and translational implications. Nat Rev Cancer 2011;11(10): 726-734.

4. Han H, Cortez CC, Yang X, Nichols PW, Jones PA, Liang G. DNA methylation directly silences genes with non-CpG island promoters and establishes a nucleosome occupied promoter. Hum Mol Genet. 2011;20(22):4299-4310.

5. Bestor TH. The DNA methyltransferases of mammals. Hum Mol Genet. 2000;9(16):2395-2402.

6. Jin B, Robertson KD. DNA methyltransferases, DNA damage repair, and cancer. Adv Exp Med Biol. 2013;754:3-29.

7. Christophorou MA, Castelo-Branco G, Halley-Stott RP, et al. Citrullination regulates pluripotency and histone $\mathrm{H} 1$ binding to chromatin. Nature. 2014;507(7490):104-108.

8. Deplus R, Denis H, Putmans P, et al. Citrullination of DNMT3A by PADI4 regulates its stability and controls DNA methylation. Nucleic Acids Res. 2014;42(13):8285-8296.

9. Agarwal S, Amin KS, Jagadeesh S, et al. Mahanine restores RASSF1A expression by down-regulating DNMT1 and DNMT3B in prostate cancer cells. Mol Cancer. 2013;12(1):99.

10. Yaqinuddin A, Qureshi SA, Qazi R, Abbas F. Down-regulation of DNMT3b in PC3 cells effects locus-specific DNA methylation, and represses cellular growth and migration. Cancer Cell Int. 2008; 8:13.

11. Donninger H, Vos MD, Clark GJ. The RASSF1A tumor suppressor. J Cell Sci. 2007;120(Pt 18):3163-3172.

12. Hesson LB, Cooper WN, Latif F. The role of RASSF1A methylation in cancer. Dis Markers. 2007;23(1-2):73-87.

13. Mengxi D, Qian W, Nan W, Xiaoguang X, Shijun L. Effect of DNA methylation inhibitor on RASSF1A genes expression in non-small cell lung cancer cell line A549 and A549DDP. Cancer Cell Int. 2013; 13(1):91.

14. Qiu X, Zhang L, Lu S, et al. Upregulation of DNMT1 mediated by HBx suppresses RASSF1A expression independent of DNA methylation. Oncol Rep. 2014;31(1):202-208.

15. Varjosalo M, Taipale J. Hedgehog: functions and mechanisms. Genes Dev. 2008;22(18):2454-2472.

16. Echelard Y, Epstein DJ, St-Jacques B, et al. Sonic hedgehog, a member of a family of putative signaling molecules, is implicated in the regulation of CNS polarity. Cell. 1993;75(7):1417-1430.

17. Chou RH, Wang YN, Hsieh YH, et al. EGFR modulates DNA synthesis and repair through Tyr phosphorylation of histone H4. Dev Cell. 2014; 30(2):224-237.

18. Shi X, Zhang Z, Zhan X, et al. An epigenetic switch induced by Shh signalling regulates gene activation during development and medulloblastoma growth. Nat Commun. 2014;5:5425.

19. Chow KH, Shin DM, Jenkins MH, et al. Epigenetic states of cells of origin and tumor evolution drive tumor-initiating cell phenotype and tumor heterogeneity. Cancer Res. 2014;74(17):4864-4874.

20. Shahi MH, Afzal M, Sinha S, et al. Regulation of sonic hedgehog-GLI1 downstream target genes PTCH1, Cyclin D2, Plakoglobin, PAX6 and NKX2.2 and their epigenetic status in medulloblastoma and astrocytoma. BMC Cancer. 2010;10:614

21. Moon RT, Kohn AD, De Ferrari GV, Kaykas A. WNT and beta-catenin signalling: diseases and therapies. Nat Rev Genet. 2004;5(9):691-701.

22. Klaus A, Birchmeier W. Wnt signalling and its impact on development and cancer. Nat Rev Cancer. 2008;8(5):387-398. 
23. Samaei NM, Yazdani Y, Alizadeh-Navaei R, Azadeh H, Farazmandfar T. Promoter methylation analysis of WNT/beta-catenin pathway regulators and its association with expression of DNMT1 enzyme in colorectal cancer. J Biomed Sci. 2014;21:73.

24. Bernstein C, Bernstein H, Payne CM, Dvorak K, Garewal H. Field defects in progression to gastrointestinal tract cancers. Cancer Lett. 2008; 260(1-2):1-10.

25. Valcz G, Patai AV, Kalmar A, et al. Myofibroblast-derived SFRP1 as potential inhibitor of colorectal carcinoma field effect. PLoS One. 2014;9(11):e106143.

26. Anastas JN, Moon RT. WNT signalling pathways as therapeutic targets in cancer. Nat Rev Cancer. 2013;13(1):11-26.

27. Silva AL, Dawson SN, Arends MJ, et al. Boosting Wnt activity during colorectal cancer progression through selective hypermethylation of Wnt signaling antagonists. BMC Cancer. 2014;14:891.

28. Xie Q, Chen L, Shan X, et al. Epigenetic silencing of SFRP1 and SFRP 5 by hepatitis $B$ virus $X$ protein enhances hepatoma cell tumorigenicity through Wnt signaling pathway. Int $J$ Cancer. 2014;135(3): 635-646.

29. Kim HY, Park JH, Won HY, Lee JY, Kong G. CBX7 inhibits breast tumorigenicity through DKK-1-mediated suppression of the Wnt/betacatenin pathway. FASEB J. 2015;29(1):300-313.

30. Yin X, Xiang T, Li L, et al. DACT1, an antagonist to Wnt/beta-catenin signaling, suppresses tumor cell growth and is frequently silenced in breast cancer. Breast Cancer Res. 2013;15(2):R23.

31. Zhao Z, Herman JG, Brock MV, et al. Methylation of DACT2 promotes papillary thyroid cancer metastasis by activating Wnt signaling. PLoS One. 2014;9(11):e112336.

32. MacDonald BT, Tamai K, He X. Wnt/beta-catenin signaling: components, mechanisms, and diseases. Dev Cell. 2009;17(1):9-26.

33. Miao Y, Wang L, Zhang X, et al. Promoter methylation-mediated silencing of beta-catenin enhances invasiveness of non-small cell lung cancer and predicts adverse prognosis. PLoS One. 2014;9(11):e112258.

34. Gong C, Qu S, Lv XB, et al. BRMS1L suppresses breast cancer metastasis by inducing epigenetic silence of FZD10. Nat Commun. 2014;5:5406.

35. Ronsch K, Jagle S, Rose K, et al. SNAIL1 combines competitive displacement of ASCL2 and epigenetic mechanisms to rapidly silence the EPHB3 tumor suppressor in colorectal cancer. Mol Oncol. 2015;9(2):335-354.

36. Tong X, Li L, Li X, et al. SOX10, a novel HMG-box-containing tumor suppressor, inhibits growth and metastasis of digestive cancers by suppressing the Wnt/beta-catenin pathway. Oncotarget. 2014; 5(21):10571-10583.

37. Zhang S, Li S, Gao JL. Promoter methylation status of the tumor suppressor gene SOX11 is associated with cell growth and invasion in nasopharyngeal carcinoma. Cancer Cell Int. 2013;13(1):109.

38. Li BL, $\mathrm{Lu} \mathrm{W}, \mathrm{Lu} \mathrm{C}$, et al. CpG island hypermethylation-associated silencing of microRNAs promotes human endometrial cancer. Cancer Cell Int. 2013;13(1):44.

39. Shiah SG, Hsiao JR, Chang WM, et al. Downregulated miR329 and miR410 promote the proliferation and invasion of oral squamous cell carcinoma by targeting Wnt-7b. Cancer Res. 2014;74(24):7560-7572.

40. Kuang SQ, Fang Z, Zweidler-McKay PA, et al. Epigenetic inactivation of Notch-Hes pathway in human B-cell acute lymphoblastic leukemia. PLoS One. 2013;8(4):e61807.

41. Groot AJ, Vooijs MA. The role of Adams in Notch signaling. Adv Exp Med Biol. 2012;727:15-36.

42. Benedito R, Roca C, Sorensen I, et al. The notch ligands D114 and Jagged1 have opposing effects on angiogenesis. Cell. 2009;137(6):1124-1135.

43. Arumugam TV, Cheng YL, Choi Y, et al. Evidence that gammasecretase-mediated Notch signaling induces neuronal cell death via the nuclear factor-kappaB-Bcl-2-interacting mediator of cell death pathway in ischemic stroke. Mol Pharmacol. 2011;80(1):23-31.

44. Miele L. Transcription factor RBPJ/CSL: a genome-wide look at transcriptional regulation. Proc Natl Acad Sci U S A. 2011;108(36): $14715-14716$.
45. Oswald F, Tauber B, Dobner T, et al. p300 acts as a transcriptional coactivator for mammalian Notch-1. Mol Cell Biol. 2001;21(22): 7761-7774.

46. Nam Y, Sliz P, Song L, Aster JC, Blacklow SC. Structural basis for cooperativity in recruitment of MAML coactivators to Notch transcription complexes. Cell. 2006;124(5):973-983.

47. Castel D, Mourikis P, Bartels SJ, Brinkman AB, Tajbakhsh S, Stunnenberg HG. Dynamic binding of RBPJ is determined by Notch signaling status. Genes Dev. 2013;27(9):1059-1071.

48. Man $\mathrm{CH}$, Wei-Man Lun S, Wai-Ying Hui J, et al. Inhibition of NOTCH3 signalling significantly enhances sensitivity to cisplatin in EBV-associated nasopharyngeal carcinoma. J Pathol. 2012;226(3): 471-481.

49. Zampieri M, Ciccarone F, Palermo R, et al. The epigenetic factor BORIS/CTCFL regulates the NOTCH3 gene expression in cancer cells. Biochim Biophys Acta. 2014;1839(9):813-825.

50. Liu Q, Zheng JM, Chen JK, et al. Histone deacetylase 5 promotes the proliferation of glioma cells by upregulation of Notch 1. Mol Med Rep. 2014;10(4):2045-2050.

51. Kong D, Heath E, Chen W, et al. Epigenetic silencing of miR-34a in human prostate cancer cells and tumor tissue specimens can be reversed by BR-DIM treatment. Am J Transl Res. 2012;4(1):14-23.

52. Liu MX, Siu MK, Liu SS, Yam JW, Ngan HY, Chan DW. Epigenetic silencing of microRNA-199b-5p is associated with acquired chemoresistance via activation of JAG1-Notch1 signaling in ovarian cancer. Oncotarget. 2014;5(4):944-958.

53. Liu Y, Xing ZB, Wang SQ, et al. MDM2-MOF-H4K16ac axis contributes to tumorigenesis induced by Notch. FEBS J. 2014;281(15): 3315-3324.

54. Weng AP, Ferrando AA, Lee W, et al. Activating mutations of NOTCH1 in human T cell acute lymphoblastic leukemia. Science. 2004; 306(5694):269-271.

55. Katoh M, Katoh M. Integrative genomic analyses on HES/HEY family: Notch-independent HES1, HES3 transcription in undifferentiated ES cells, and Notch-dependent HES1, HES5, HEY1, HEY2, HEYL transcription in fetal tissues, adult tissues, or cancer. Int $J$ Oncol. 2007;31(2):461-466.

56. Choi JH, Park JT, Davidson B, Morin PJ, Shih Ie M, Wang TL. Jagged-1 and Notch3 juxtacrine loop regulates ovarian tumor growth and adhesion. Cancer Res. 2008;68(14):5716-5723.

57. Ikushima H, Miyazono K. TGFbeta signalling: a complex web in cancer progression. Nat Rev Cancer. 2010;10(6):415-424.

58. Massague J. TGFbeta signalling in context. Nat Rev Mol Cell Biol.2012; 13(10):616-630.

59. Qian Q, Shi X, Lei Z, et al. Methylated $+58 \mathrm{CpG}$ site decreases DCN mRNA expression and enhances TGF-beta/Smad signaling in NSCLC cells with high metastatic potential. Int J Oncol. 2014; 44(3):874-882.

60. Chan MW, Huang YW, Hartman-Frey C, et al. Aberrant transforming growth factor beta1 signaling and SMAD4 nuclear translocation confer epigenetic repression of ADAM19 in ovarian cancer. Neoplasia. 2008;10(9):908-919.

61. Wang A, Pan D, Lee YH, Martinez GJ, Feng XH, Dong C. Cutting edge: Smad2 and Smad4 regulate TGF-beta-mediated I19 gene expression via EZH2 displacement. J Immunol. 2013;191(10):4908-4912.

62. Guo W, Zhang M, Shen S, et al. Aberrant methylation and decreased expression of the TGF-beta/Smad target gene FBXO32 in esophageal squamous cell carcinoma. Cancer. 2014;120(16):2412-2423.

63. Qin H, Qu C, Yamaza T, et al. Ossifying fibroma tumor stem cells are maintained by epigenetic regulation of a TSP1/TGF-beta/SMAD3 autocrine loop. Cell Stem Cell. 2013;13(5):577-589.

64. Zhu H, Wu K, Yan W, et al. Epigenetic silencing of DACH1 induces loss of transforming growth factor-beta 1 antiproliferative response in human hepatocellular carcinoma. Hepatology. 2013;58(6):2012-2022.

65. Yan W, Wu K, Herman JG, et al. Epigenetic silencing of DACH1 induces the invasion and metastasis of gastric cancer by activating TGF-beta signalling. J Cell Mol Med. 2014;18(12):2499-2511. 
66. Cardenas H, Vieth E, Lee J, et al. TGF-beta induces global changes in DNA methylation during the epithelial-to-mesenchymal transition in ovarian cancer cells. Epigenetics. 2014;9(11):1461-1472.

67. Kim HJ, Kim JH, Chie EK, Young PD, Kim IA, Kim IH. DNMT (DNA methyltransferase) inhibitors radiosensitize human cancer cells by suppressing DNA repair activity. Radiat Oncol. 2012;7:39.

68. Huang YC, Chao KS, Liao HF, Chen YJ. Targeting sonic hedgehog signaling by compounds and derivatives from natural products. Evid Based Complement Alternat Med. 2013;2013:748587.

69. Zhang J, Kong LM, Zhan R, et al. Two natural ent-kauranoids as novel Wnt signaling inhibitors. Nat Prod Bioprospect. 2014;4(3):135-140.

70. Kang YJ, Park HJ, Chung HJ, et al. Wnt/beta-catenin signaling mediates the antitumor activity of magnolol in colorectal cancer cells. Mol Pharmacol. 2012;82(2):168-177.

71. Lee C, Zhang Q, Kozlowski J, et al. Natural products and transforming growth factor-beta (TGF-beta) signaling in cancer development and progression. Curr Cancer Drug Targets. 2013;13(5):500-505.

72. Choi JH, Hwang YP, Kim HG, et al. Saponins from the roots of Platycodon grandiflorum suppresses TGFbeta1-induced epithelialmesenchymal transition via repression of PI3K/Akt, ERK1/2 and Smad2/3 pathway in human lung carcinoma A549 cells. Nutr Cancer. 2014;66(1):140-151.

73. Jia L, Jin H, Zhou J, et al. A potential anti-tumor herbal medicine, Corilagin, inhibits ovarian cancer cell growth through blocking the TGF-beta signaling pathways. BMC Complement Altern Med. 2013;13:33.

74. Kim SO, Kim MR. (-)-Epigallocatechin 3-gallate inhibits invasion by inducing the expression of Raf kinase inhibitor protein in AsPC1 human pancreatic adenocarcinoma cells through the modulation of histone deacetylase activity. Int J Oncol. 2013;42(1):349-358.

75. Groh IA, Chen C, Luske C, Cartus AT, Esselen M. Plant polyphenols and oxidative metabolites of the herbal alkenylbenzene methyleugenol suppress histone deacetylase activity in human colon carcinoma cells. J Nutr Metab. 2013;2013:821082.
76. Wang H, Li Q, Chen H. Genistein affects histone modifications on Dickkopf-related protein 1 (DKK1) gene in SW480 human colon cancer cell line. PLoS One. 2012;7(7):e40955.

77. Baud MG, Leiser T, Haus $P$, et al. Defining the mechanism of action and enzymatic selectivity of psammaplin A against its epigenetic targets. J Med Chem. 2012;55(4):1731-1750.

78. Naselli F, Tesoriere L, Caradonna F, et al. Anti-proliferative and pro-apoptotic activity of whole extract and isolated indicaxanthin from Opuntia ficus-indica associated with re-activation of the oncosuppressor p16(INK4a) gene in human colorectal carcinoma (Caco-2) cells. Biochem Biophys Res Commun. 2014;450(1):652-658.

79. Su ZY, Khor TO, Shu L, et al. Epigenetic reactivation of Nrf2 in murine prostate cancer TRAMP C1 cells by natural phytochemicals Z-ligustilide and Radix angelica sinensis via promoter $\mathrm{CpG}$ demethylation. Chem Res Toxicol. 2013;26(3):477-485.

80. Wu FL, Li RT, Yang M, et al. Gelatinases-stimuli nanoparticles encapsulating 5-fluorouridine and 5-aza-2'-deoxycytidine enhance the sensitivity of gastric cancer cells to chemical therapeutics. Cancer Lett. 2015: in press [DOI: 10.1016/j.canlet.2015.1001.1006].

81. Su X, Wang Z, Li L, et al. Lipid-polymer nanoparticles encapsulating doxorubicin and 2 '-deoxy-5-azacytidine enhance the sensitivity of cancer cells to chemical therapeutics. Mol Pharm. 2013;10(5):1901-1909.

82. Legut M, Lipka D, Filipczak N, Piwoni A, Kozubek A, Gubernator J. Anacardic acid enhances the anticancer activity of liposomal mitoxantrone towards melanoma cell lines - in vitro studies. Int J Nanomedicine. 2014;9:653-668.

83. Meng J, Xing J, Wang Y, et al. Epigenetic modulation of human breast cancer by metallofullerenol nanoparticles: in vivo treatment and in vitro analysis. Nanoscale. 2011;3(11):4713-4719.

84. Li SY, Sun R, Wang HX, et al. Combination therapy with epigenetictargeted and chemotherapeutic drugs delivered by nanoparticles to enhance the chemotherapy response and overcome resistance by breast cancer stem cells. J Control Release. 2014: in press [DOI: 10.1016/j. jconrel.2014.1011.1011].
International Journal of Nanomedicine

\section{Publish your work in this journal}

The International Journal of Nanomedicine is an international, peerreviewed journal focusing on the application of nanotechnology in diagnostics, therapeutics, and drug delivery systems throughou the biomedical field. This journal is indexed on PubMed Central,

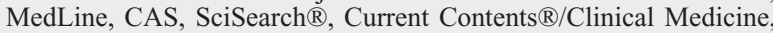

\section{Dovepress}

Journal Citation Reports/Science Edition, EMBase, Scopus and the Elsevier Bibliographic databases. The manuscript management system is completely online and includes a very quick and fair peer-review system, which is all easy to use. Visit http://www.dovepress.com/ testimonials.php to read real quotes from published authors. 